\title{
Manipulation of magnetization in GaMnAs films by spin-orbit-induced
}

\section{magnetic fields}

\author{
Sangyeop Lee ${ }^{1}$, Taehee Yoo ${ }^{1,2}$, Seul-Ki Bac ${ }^{1}$, Seonghoon Choi ${ }^{1}$, Hakjoon Lee ${ }^{1}$, \\ Sanghoon Lee ${ }^{1 *}$, X. Liu $^{2}$, J. K. Furdyna ${ }^{2}$, and M. Dobrowolska ${ }^{2}$ \\ ${ }^{1}$ Physics Department, Korea University, Seoul 136-701, Korea \\ ${ }^{2}$ Physics Department, University of Notre Dame, Notre Dame, IN 46556, USA
}

We have investigated the effect of spin-orbit-induced (SOI) magnetic fields on magnetization switching in GaMnAs films. The sign of such SOI fields depends on the direction of the current flowing in the film, thus providing a handle for electrically manipulating magnetization in ferromagnetic GaMnAs films. Specifically, when an applied magnetic field is swept along the current direction, magnetization reversal occurs via rotations in opposite sense (i.e., clockwise (CW) or counterclockwise (CCW)) depending on the sign of the current, thus leading to opposite signs of the planar Hall resistance (PHR) measured on the film. The effect of SOI fields also manifests itself through hysteretic behavior of PHR for two opposite currents as a fixed magnetic field is rotated in the film plane. The width of the resulting hysteresis between two current directions then allows us to estimate the magnitude of the SOI field at current density of $1.0 \times 10^{5} \mathrm{~A} / \mathrm{cm}^{2}$ as $\sim 1.2$ Oe in our GaMnAs film. Such switching of magnetization between two magnetic easy axes induced by switching the sign of an applied current provides a means of electronically controlling the value of film resistance (in this case of PHR), a process that can be exploited in spintronic devices.

PACS number: 71.70.Ej, 72.80.Ey, 75.30.Gw, 75.47.-m, 75.70.Ak

Keywords : Spin orbit induced field, Planar Hall resistance, Ferromagnetic semiconductors Email : slee3@korea.ac.kr

Fax: +82-2-920-3592 


\section{Introduction}

Manipulating the direction of magnetization in ferromagnetic (FM) materials is of key importance for operating spintronic devices, in which information is stored and processed through magnetization orientation. The classical method of using an external magnetic field for this purpose is now increasingly being replaced by the technique referred to as spin transfer torque (STT) generated by a spin-polarized electric current passing through a device.[1-3] Since the STT approach provides significant advantages in simplifying and miniaturizing the device structure, and in substantially increasing its operating speed, STT has been intensively investigated in magnetic systems, especially in the past decade. [4-6]

Although the STT approach has already been integrated into several types of spintronic devices, such as STT-based magnetoresistive random access memories (MRAMs),[7-10] there exists continued demand for reducing the current densities used for manipulating magnetization in such devices. Recently, and important advance has been made in this context when it was discovered that current-induced magnetic fields originating from spin-orbit interaction (SOI) can be used for manipulating magnetization in ferromagnetic films. [11, 12] It is well known that the broken inversion symmetry in certain crystal structures as well as the strain distorting crystalline bonds are the two major mechanisms for such SOI fields in magnetic films.[11, 13, 14] Ferromagnetic GaMnAs films, in which structural inversion symmetry is broken, and which are under compressive strain due to lattice mismatch with GaAs substrates on which they are typically grown, are thus ideally suited for investigating such SOI fields.

In this paper we study the influence of the SOI field on magnetization reversal in a GaMnAs film, and we demonstrate that its magnetization can be manipulated electric currents which produce these fields. We identify the presence of such SOI fields in the GaMnAs film by using planar Hall resistance (PHR) measurements performed in two ways. First, we carry out experiments where the magnetization of the GaMnAs film is reversed by sweeping a magnetic field applied at a fixed angle near the hard magnetic axis of the FM film. In a second set of experiments we rotate a field with a fixed magnitude over $360^{\circ}$ in both clockwise $(\mathrm{CW})$ and counterclockwise $(\mathrm{CCW})$ directions, and measure PHR in the presence of currents with opposite polarity in the GaMnAs device. These experiments allow us to 
optimize conditions for manipulating the orientation of magnetization in the GaMnAs Hall device by SOI fields as one switches the sign of the current flowing in the device.

\section{Sample preparation and experiments}

The GaMnAs film used in this investigation was grown by molecular beam epitaxy (MBE) on a (001) GaAs substrate. Prior to the growth of the GaMnAs layer, a $100 \mathrm{~nm}$ GaAs buffer layer was deposited on the substrate at $600{ }^{\circ} \mathrm{C}$, followed by deposition of a $2 \mathrm{~nm}$ of GaAs layer at $250{ }^{\circ} \mathrm{C}$. An $100 \mathrm{~nm} \mathrm{Ga}{ }_{1-x} \mathrm{Mn}_{x}$ As film with $x=0.08$ was then grown on top of the GaAs buffer. For investigating the effects of SOI fields on the orientation of magnetization, we have chosen to use the planar Hall effect, which is especially sensitive to the direction of magnetization. For these measurements, a $5 \times 5 \mathrm{~mm}^{2}$ piece was cut from the GaMnAs wafer, and a Hall device was fabricated by photolithography and dry etching in the shape of a strip $1000 \mu \mathrm{m}$ long and $10 \mu \mathrm{m}$ wide, with the long dimension along the [1 10$]$ crystalline direction, as schematically shown in Fig. 1(a). In this paper we will refer to the current flowing along the [1 $\overline{1} 0]$ directions as positive current, and to current along [110] as negative.

The PHR measurements were performed using a sample holder designed so as to allow a magnetic field to be applied at arbitrary directions in the plane of the sample. The electromagnet used for this purpose was mounted on a rotating table, so that the field could either be swept along an arbitrary fixed direction, or could be continuously rotated in the film plane with a fixed magnitude. The directions of the applied magnetic field $\varphi_{H}$ and of the magnetization of the GaMnAs film $\varphi_{M}$ are measured in the (001) crystal plane,

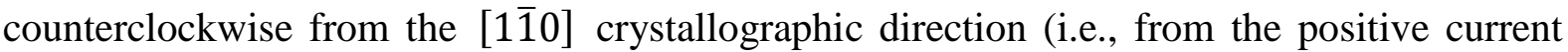
direction of in the Hall device).

The temperature of the sample holder was set at $3 \mathrm{~K}$ during the measurements. However, it is known that Joule heating by the current flowing in the device changes its temperature during the measurement. $[15,16]$ The current of $\pm 1 \mathrm{~mA}$, which corresponds to a current density of $1.0 \times 10^{5} \mathrm{~A} / \mathrm{cm}^{2}$ used in this study, is indeed sufficiently large to generate significant Joule heating in our Hall device. The magnetic anisotropy of the GaMnAs film, which governs the process of magnetization reversal, is then expected to change due to the increased temperature of the device. We have therefore investigated the magnetic anisotropy 
of the GaMnAs film in the presence of the same current of $1.0 \times 10^{5} \mathrm{~A} / \mathrm{cm}^{2}$ as that used for manipulating magnetization in experiments discussed below. The shape of the magnetic free energy profile obtained for our GaMnAs film in the presence of this current of this is shown in Fig. 1(b). Magnetization switching in the GaMnAs film observed in our experiments will then be determined by this magnetic energy density profile.

\section{Results and Discussion}

The presence of the SOI field in our GaMnAs film was first tested by measuring the PHR using positive and negative currents during magnetization reversal, as follows. The field orientation was set along the [1ㅣㅣㄹ hard axis (i.e., $\varphi_{H}=0^{\circ}$ ), so that the symmetry of the magnetic energy profile with respect to this axis is not affected as the field is swept. The rotation of magnetization direction $(\mathrm{CW}$ or $\mathrm{CCW})$ during the reversal process will then be dictated only by the direction of the SOI field associated with the direction of the current flowing through the device. To see this, PHR was measured for two opposite current directions as the field was swept from -1500 Oe to +1500 Oe. The PHR data obtained during this field sweep are shown in Fig. 2(a), in which we only plot data for the field region between -200 Oe and +200 Oe. Here red circles correspond to data taken with positive current (i.e., flowing along [1 $1 \overline{1} 0]$ ), and blue squares are for negative current (i.e., along [110]). The PHR data shows a clear difference for the two current directions, which can be understood through a difference in magnetization relaxation due to different SOI fields induced by the two currents. When a strong magnetic field (i.e., in the range between $-1,500$ Oe and $-200 \mathrm{Oe})$ is applied along the [110] direction, the lowest magnetic energy minimum lies along that direction due to the dominance of Zeeman energy, and the magnetization will be aligned with this energy minimum. This is shown by the set of insets on the leftmost side of Fig. 2(a), where the direction of magnetization is expressed as a thick solid arrow in the polar plot. Such direction of magnetization can be identified from PHR using the relation [17]

$R_{P H E}=\frac{k}{t} M^{2} \sin 2 \varphi_{M}$

where $k$ is a constant related to the anisotropic magnetoresistance; $M$ is the magnetization of the film; $t$ is its thickness; and $\varphi_{M}$ is the direction of the magnetization as defined in Fig. 1(a). Since the magnetization points in the $[\overline{1} 10]$ direction (i.e., $\varphi_{H}=180^{\circ}$ ) under a strong 
field, the value of PHR is nearly zero, as seen in the data in Fig. 2(a).

When the field strength decreases (from negative fields toward zero), the magnetization in the GaMnAs film begins to relax from the [110] direction toward either [110] or [ $\overline{1} \overline{1} 0]$. The change of the direction of magnetization is reflected in PHR according to Eq. (1). PHR values measured for two opposite current directions deviate from each other in fields weaker than -200 Oe, as seen in Fig. 2(a). Note that PHR tends to negative values for a positive current (red points), and to positive values for a negative current (blue points). This indicates that, as the field strength is reduced, the magnetization of the GaMnAs film rotates

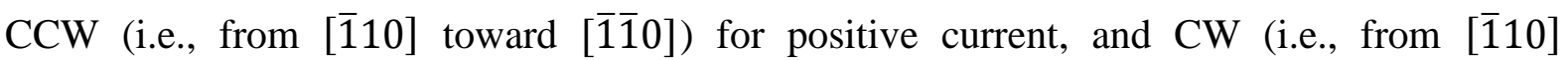
toward [110]) for negative current. This process is schematically illustrated in the figure with insets at successive field positions. Such process of magnetization relaxation indicates that

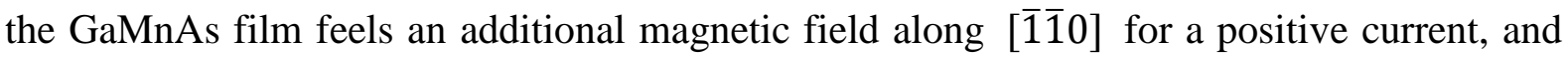
along [110] for a negative current. The direction of this additional magnetic field is the same as that of the SOI field caused by strain, as shown in Fig. 1(b) (red arrows). This observation is consistent with earlier investigations, which reported that in GaMnAs films the SOI fields induced by strain are about three times stronger than those arising from broken inversion symmetry (i.e., Rashba-like fields).[13]

The magnetization relaxation seen in Fig. 2(a) can be more clearly understood when the effect of the SOI field is included in the energy profile, as shown in Fig. 2(b)-(e). The effect of the SOI field is undetectable when the applied magnetic field is strong, as is the case for either -200 Oe or +200 Oe in Fig. 2(b) and 2(e). In that strong field limit the energy profiles obtained for two opposite current directions nearly overlap with one another, showing a single minimum for the $[\overline{1} 10]$ or the [1ํㅣㄹ direction (i.e., the field direction) due to the dominance of the Zeeman energy term. As the field strength is reduced, however, magnetic energy profiles for the two current directions clearly deviate from each other. For example, at -25 Oe the energy minimum is lower at [100] than at [010] for a positive current; and the opposite is true for a negative current, as shown in Fig. 2(c). This is entirely due to the effect of SOI fields, which are along the [1]10] direction for the positive, and along the [110] for negative currents. The magnetization then relaxes toward the lower energy minimum, leading to a CCW rotation (from [110]) toward [100]) for positive currents, and to a $\mathrm{CW}$ rotation (from [110] to [010]) for negative currents, as illustrated in the left side of Fig. 2(a). 
Once the magnetization of the film starts to relax via either $\mathrm{CCW}$ or $\mathrm{CW}$ rotation, it continuous to follow the position of the energy minimum as the field is reduced. Thus, when

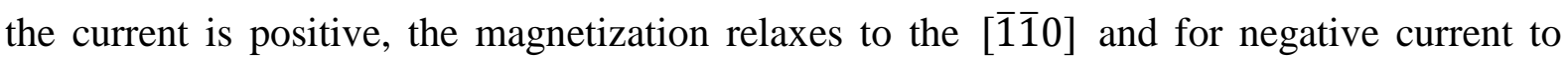
[110], the two magnetic energy minima at zero field shown in Fig. 1(b). This is seen in the data shown in Fig. 2(a), where PHR measured with the two current directions are clearly different, and cross at zero field. When the magnetic field is swept to positive direction and its strength is increased, the magnetization begins to rotate toward the [1ํㅣ direction (i.e., to the field direction) for both positive and negative currents. The direction of magnetization then passes the [010] direction for positive current, and the [100] direction for negative current, reaching the [1]10] direction at strong fields when the magnetization reversal process is completed (around 200 Oe). Such dependence of magnetization relaxation on current direction indicates the presence of SOI fields in our GaMnAs film.

The presence of SOI field is further confirmed by the angular dependence of PHR observed by rotating the external field direction $\varphi_{H}$ in the sample plane at constant field strength. For this measurement, the magnetization of the film was initialized to the energy minimum in the $4^{\text {th }}$ quadrant using a field of 2000 Oe applied along $\varphi_{H}=-60^{\circ}$. The field was then reduced to $15 \mathrm{Oe}$, and PHR was measured with currents of +1.0 and $-1.0 \mathrm{~mA}$ at different angles as the field was rotated between $\varphi_{H}=-50^{\circ}$ and $\varphi_{H}=420^{\circ}$. The results are plotted in Fig. 3, where open and solid symbols are used for PHR data obtained with CW and CCW rotation of the field, squares and triangles representing values obtained with positive and negative currents, respectively. Note that at [110] and [110] directions the PHR data show clear hysteresis between the positive and the negative currents. Such hysteresis can be understood by considering SOI fields that are oppositely oriented for the two currents. Specifically, when a field of 15 Oe rotates CCW across the [1ํㅣㄹ direction, a positive current generates an SOI field along the [11ㅣㄹ direction, which opposes the rotation of magnetization from the energy minimum in the $4^{\text {th }}$ quadrant to the energy minimum in the $1^{\text {st }}$ quadrant. A negative current, on the other hand, generates an SOI field along the [110] direction that assists such CCW rotation of magnetization. Thus the transition occurs at a smaller field angle (i.e., earlier) for the negative current than for the positive current when the field is rotated CCW across the [1 $\overline{1} 0]$ direction, as seen in the data plotted with solid symbols in Fig. 3.

The situation is opposite for the CCW rotation near $\varphi_{H}=180^{\circ}$, where magnetization 
crosses the [110] energy barrier. In that case the transition of magnetization across [110] occurs earlier for the positive current than for the negative current, as seen from the transitions appearing at $\varphi_{H}=180^{\circ}$ in Fig. 3 (see solid symbols). This is again due to the

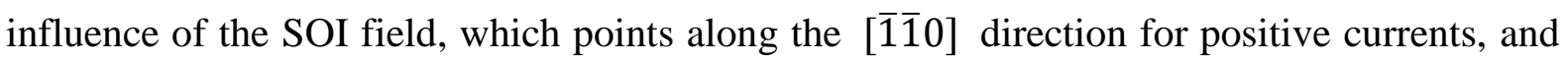
along [110] for negative currents. The SOI field along [110] then makes the CCW rotation of the magnetization over the [110] direction harder, while the SOI field along [ $\overline{1} \overline{1} 0]$ makes this rotation easier. A complimentary situation occurs when the field is rotated in the CW sense, as shown by open symbols in Fig. 3, which can be discussed following analogous steps. The strength of the SOI field can then be estimated from the difference of transition angles (i.e., from the width of the hysteresis) using the equation developed in Ref.11[11]. In our case, the effective SOI field was obtained as $\mathrm{H}_{\mathrm{SOI}}=1.2$ Oe for current density of $1.0 \times 10^{5} \mathrm{~A} / \mathrm{cm}^{2}$. The magnitude of the SOI field obtained in the present work agrees well with the values obtained for GaMnAs films in earlier studies. [11, 13, 14]

The hysteresis formed by the difference in transition angles between corresponding to positive and negative currents around the $\varphi_{H}=0^{\circ}$ and $180^{\circ}$ (i.e., around the [1 $\left.\overline{1} 0\right]$ and the [110] directions) provides an opportunity for manipulating PHR between two values by switching current directions. To test such switching process, we use the hysteresis appearing near the [1ํㅣㄹ direction in Fig. 4(a). We first set the magnetization of the GaMnAs film at the energy minimum in the $4^{\text {th }}$ quadrant (i.e., near [010]) by using a field of 2,000 Oe. The applied field is then reduced to $15 \mathrm{Oe}$ and rotated $\mathrm{CCW}$ to the [1]10] direction (i.e., $\varphi_{H}=0^{\circ}$ ), at which the 15 Oe field is kept as the background field. PHR measured with +1.0 $\mathrm{mA}$ during this preparation process is plotted as large open circles in Fig. 4(a), showing a nice overlap with previously taken angle-dependent PHR data for the CCW rotation.

The presence of the 15 Oe background field in the [1ํㅣㄹ direction decreases the height of the energy barrier, making the transition of the magnetization across the [1 $1 \overline{1} 0]$ direction easier. We then measure the PHR at this initialized state with the positive current of $+1.0 \mathrm{~mA}$ for 80 seconds, as shown by large open circles in Fig. 4 (b). The PHR is maintained at a constant value near $+0.3 \Omega$, the same as its value at the final stage of the initializing process, marked with dashed black arrow, indicating the stability of the initialized magnetic state in the GaMnAs film. After confirming the stability of this state, the polarity of the current was switched from positive to negative. As soon as the polarity of the current is reversed, the PHR makes an abrupt transition to a negative value (in this case to $-0.15 \Omega$ ), 
that corresponds to the magnetization in the $1^{\text {st }}$ quadrant. The $-0.15 \Omega$ is the same PHR value that was observed at $\varphi_{H}=0^{\circ}$ in CCW rotation in the presence of a negative current (see the position marked with blue dotted arrow from Fig. 4(b) to 4(a)). This PHR remains constant for the measurement duration of 3 minutes, until the polarity of current is switched. When the polarity of the current is changed back to positive, the PHR again makes an abrupt transition to the value of $+0.15 \Omega$ that was observed at $\varphi_{H}=0^{\circ}$ in $\mathrm{CW}$ rotation with positive current (see the position marked with red dotted arrow in Fig. 4(b) and 4(a)). The PHR value of $+0.15 \Omega$ corresponds to the magnetization along the energy minimum in the $4^{\text {st }}$ quadrant. We have repeated the change of current sign several times to test the reproducibility of such transitions. The PHR value is switched between $+0.15 \Omega$ and $-0.15 \Omega$ as the current changes sign, as seen in Fig. 4(b), maintaining a constant value until the current polarity is changed again. This indicates that the SOI field caused by the current switches the magnetization of the GaMnAs film between stable energy minima in the $1^{\text {st }}$ and in the $4^{\text {th }}$ quadrants. Our results demonstrate that the orientation of magnetization in a GaMnAs film can be efficiently controlled by current, using the dependence of SOI fields on current direction.

\section{Summary and Conclusions}

We have investigated the effect of SOI fields on the process of magnetization reversal in a GaMnAs film by using planar Hall effect measurements. The presence of an SOI field was first identified using field sweep measurements, in which magnetization is seen to relax in opposite directions for opposite directions of current flow. The effect of the SOI field was further observed in angle-dependent PHR measurements in the form of hystereses between two opposite current directions at the [1ํㅣㄹ and [110] field orientations. The geometry of our measurements clearly indicate that the dominant mechanism in generating the observed SOI fields arises from the distortion of the zinc blende lattice by strain within the GaMnAs film. The widths of the hysteresis observed during rotation of applied magnetic field allowed us to estimate the magnitude of the SOI field as $1.2 \mathrm{Oe}$ at the current density of $1.0 \times 10^{5}$ $\mathrm{A} / \mathrm{cm}^{2}$ used in our measurements. These experiments demonstrate that such current-induced SOI field can be used to switch magnetization directions, and thus to achieve ultra-fast switching between different resistance states in a Hall device. 


\section{Acknowledgments}

This research was supported by Basic Science Research Program through the National Research Foundation of Korea (NRF) funded by the Ministry of Education (2015R1D1A1A01056614); by Korea University through a grant; and by the National Science Foundation Grant DMR 1400432.

\section{Figures}

Figure 1.
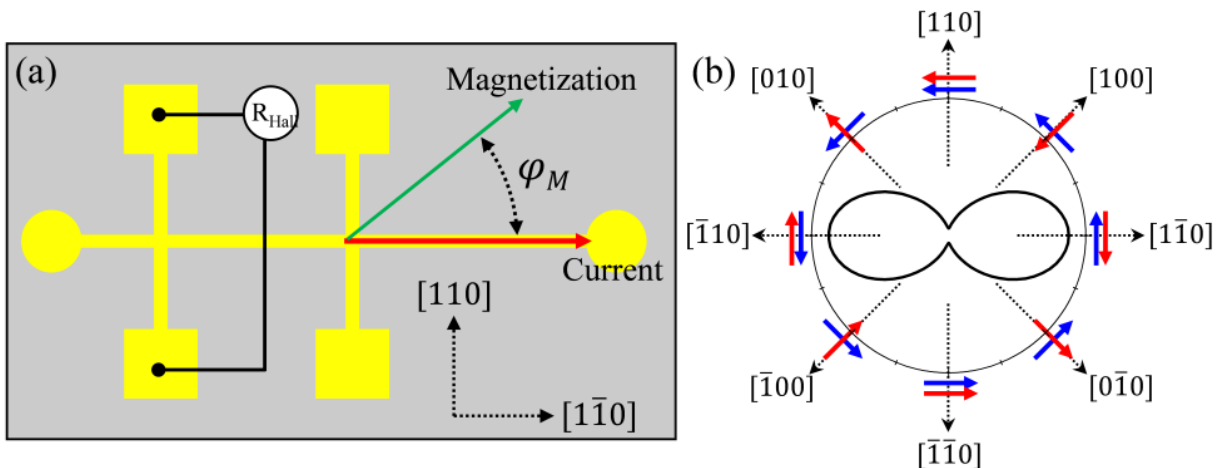

Fig. 1. (a) Schematic diagram of the Hall device used for PHR measurements patterned on a GaMnAs film. Directions of positive current and positive magnetization are shown by arrows. (b) Polar plot of the magnetic free energy density obtained for our GaMnAs film by using a current density of $1.0 \times 10^{5} \mathrm{~A} / \mathrm{cm}^{2}$. Directions of the SOI fields originating from broken inversion symmetry of GaMnAs structure are shown by blue arrows, and SOI fields originating from lattice strain are as red arrows for current directions indicated by dotted arrows. 
Figure 2.
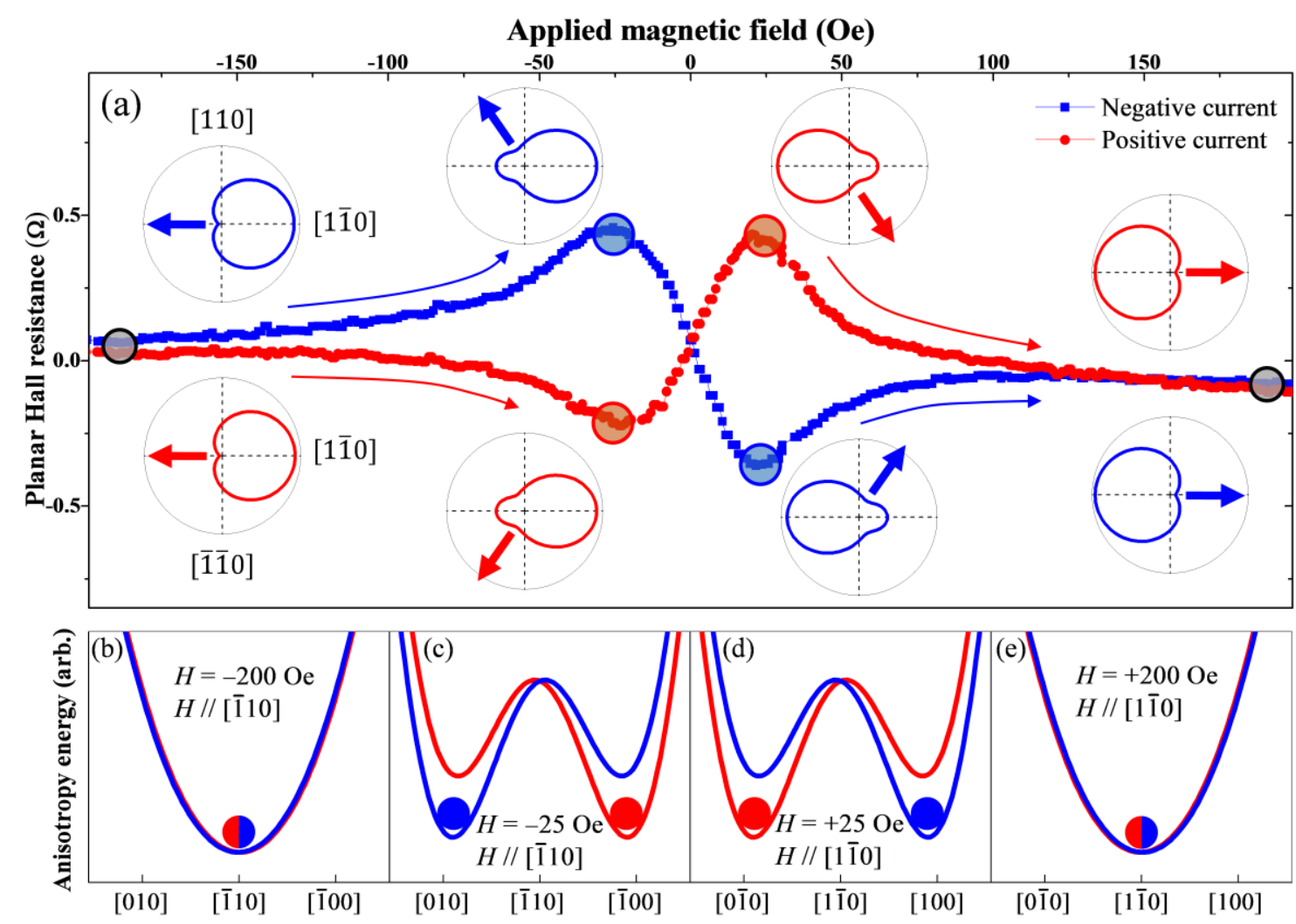

Fig. 2. (a) PHR data observed during magnetization reversal. Red circles and blue squares represent data taken with currents in positive and negative current directions, respectively. The insets show magnetic energy profiles and magnetization directions as polar plots at corresponding positions marked with small circles. (b)-(e) Magnified magnetic energy profiles (including SOI field contributions induced by current) near [1]10] field orientation at (b) -200 Oe and (c) -25 Oe, and near the [110] orientation at (d) 25 Oe and (e) at 200 Oe. Magnetic energy profiles for positive and the negative currents in (b)-(e) are shown in red and blue, respectively. 
Figure 3.

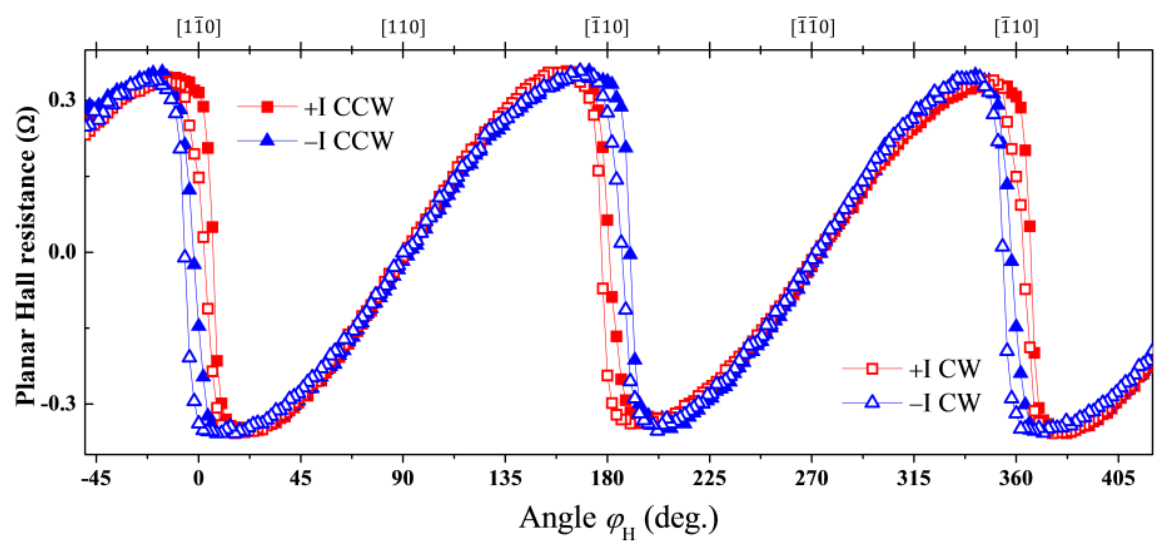

Fig.3. Angular dependence of PHR measured with two opposite current directions as the applied field is rotated $\mathrm{CW}$ and $\mathrm{CCW}$. Open symbols are used for data obtained with $\mathrm{CW}$ rotation; solid symbols for CCW rotation; squares correspond to positive current; triangles to negative current. The data show clear hystereses between $\mathrm{CW}$ and $\mathrm{CCW}$ rotations of the field as well as between positive and negative currents at $\varphi_{H}=0^{\circ}$ and $180^{\circ}$.

Figure 4. 


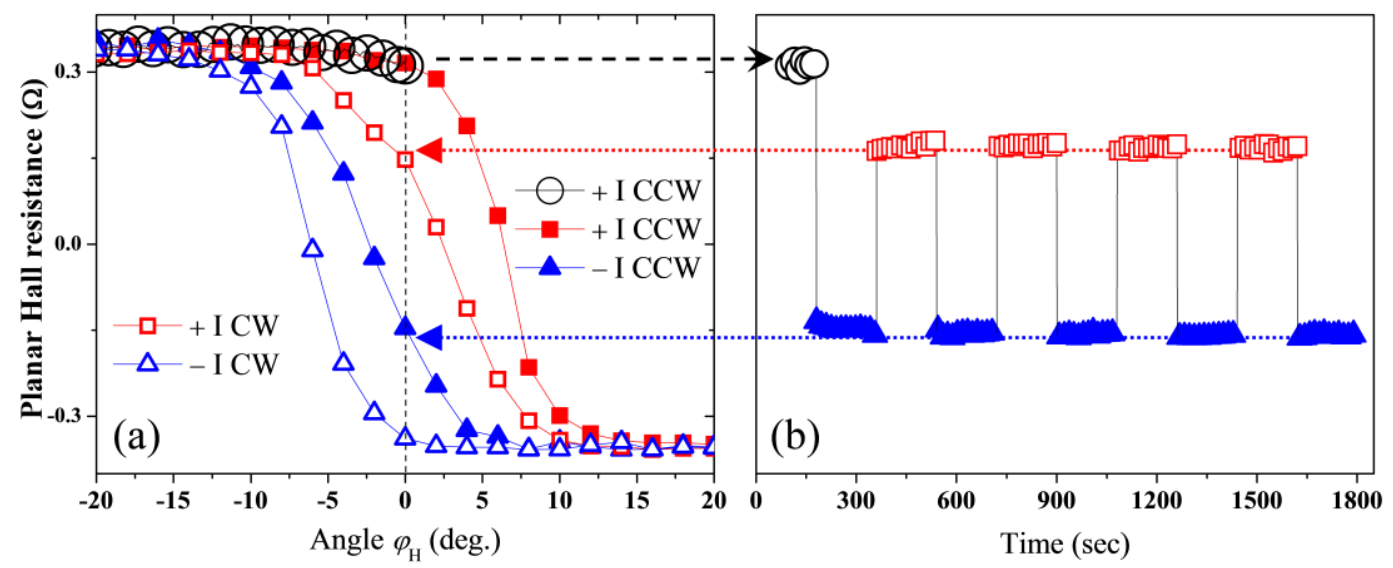

Fig. 4. (a) Magnified plot of angular dependence of PHR shown in Fig. 3 for $\varphi_{H}$ between $-20^{\circ}$ to $+20^{\circ}$. Large open circles are data obtained during the setting process, as described in the text. (b) PHR measured with two opposite current directions as a function of time, showing switching of PHR as current polarity changes.

\section{References}

[1] E.B. Myers, D.C. Ralph, J.A. Katine, R.N. Louie, R.A. Buhrman, Current-induced switching of domains in magnetic multilayer devices, Science, 285 (1999) 867-870.

[2] J.E. Wegrowe, D. Kelly, Y. Jaccard, P. Guittienne, J.P. Ansermet, Current-induced magnetization reversal in magnetic nanowires, Europhys. Lett., 45 (1999) 626-632.

[3] D.C. Ralph, M.D. Stiles, Spin transfer torques, J. Magn. Magn. Mater., 320 (2008) 1190-1216.

[4] L.Q. Liu, C.F. Pai, Y. Li, H.W. Tseng, D.C. Ralph, R.A. Buhrman, Spin-Torque Switching with the Giant Spin Hall Effect of Tantalum, Science, 336 (2012) 555-558.

[5] A.R. Mellnik, J.S. Lee, A. Richardella, J.L. Grab, P.J. Mintun, M.H. Fischer, A. Vaezi, A. Manchon, E.A. Kim, N. Samarth, D.C. Ralph, Spin-transfer torque generated by a topological insulator, Nature, 511 (2014) 449-+.

[6] S.M. Mohseni, S.R. Sani, J. Persson, T.N.A. Nguyen, S. Chung, Y. Pogoryelov, P.K. Muduli, E. Iacocca, A. Eklund, R.K. Dumas, S. Bonetti, A. Deac, M.A. Hoefer, J. Akerman, Spin TorqueGenerated Magnetic Droplet Solitons, Science, 339 (2013) 1295-1298.

[7] E. Chen, D. Apalkov, Z. Diao, A. Driskill-Smith, D. Druist, D. Lottis, V. Nikitin, X. Tang, S. Watts, 
S. Wang, S.A. Wolf, A.W. Ghosh, J.W. Lu, S.J. Poon, M. Stan, W.H. Butler, S. Gupta, C.K.A. Mewes, T. Mewes, P.B. Visscher, Advances and Future Prospects of Spin-Transfer Torque Random Access Memory, IEEE Trans. Magn., 46 (2010) 1873-1878.

[8] Z.T. Diao, Z.J. Li, S.Y. Wang, Y.F. Ding, A. Panchula, E. Chen, L.C. Wang, Y.M. Huai, Spintransfer torque switching in magnetic tunnel junctions and spin-transfer torque random access memory, J. Phys.: Condens. Matter., 19 (2007).

[9] M. Hosomi, H. Yamagishi, T. Yamamoto, K. Bessho, Y. Higo, K. Yamane, H. Yamada, M. Shoji, H. Hachino, C. Fukumoto, H. Nagao, H. Kano, A Novel Nonvolatile Memory with Spin Torque Transfer Magnetization Switching:Spin-RAM International Electron Devices Meeting, (2005).

[10] D. Apalkov, A. Khvalkovskiy, S. Watts, V. Nikitin, X.T. Tang, D. Lottis, K. Moon, X. Luo, E. Chen, A. Ong, A. Driskill-Smith, M. Krounbi, Spin-Transfer Torque Magnetic Random Access Memory (STT-MRAM), Acm J Emerg Tech Com, 9 (2013).

[11] A. Chernyshov, M. Overby, X.Y. Liu, J.K. Furdyna, Y. Lyanda-Geller, L.P. Rokhinson, Evidence for reversible control of magnetization in a ferromagnetic material by means of spin-orbit magnetic field, Nature Phys., 5 (2009) 656-659.

[12] I.M. Miron, G. Gaudin, S. Auffret, B. Rodmacq, A. Schuhl, S. Pizzini, J. Vogel, P. Gambardella, Current-driven spin torque induced by the Rashba effect in a ferromagnetic metal layer, Nature Mater., 9 (2010) 230-234.

[13] Y.Y. Li, Y.F. Cao, G.N. Wei, Y.Y. Li, Y. Ji, K.Y. Wang, K.W. Edmonds, R.P. Campion, A.W. Rushforth, C.T. Foxon, B.L. Gallagher, Anisotropic current-controlled magnetization reversal in the ferromagnetic semiconductor (Ga,Mn)As, Appl. Phys. Lett., 103 (2013).

[14] M. Endo, F. Matsukura, H. Ohno, Current induced effective magnetic field and magnetization reversal in uniaxial anisotropy (Ga,Mn)As, Appl. Phys. Lett., 97 (2010).

[15] J. Curiale, A. Lemaitre, T. Niazi, G. Faini, V. Jeudy, Joule heating and current-induced domain wall motion, J. Appl. Phys., 112 (2012).

[16] A. Yamaguchi, S. Nasu, H. Tanigawa, T. Ono, K. Miyake, K. Mibu, T. Shinjo, Effect of Joule heating in current-driven domain wall motion, Appl. Phys. Lett., 86 (2005). 
[17] K. Okamoto, A new method for analysis of magnetic anisotropy in films using the spontaneous Hall effect, J. Magn. Magn. Mater., 35 (1983) 353-355. 